\title{
Water-soluble Fluorescent Nanobowls Constructed by Multiple Supramolecular
}

\section{Assembly}

Jin Huang, * Linlin Su, Yixiao Hang, Binbin Shi, Xiaodong Wang and Hui Xu*

Institute of Advanced Synthesis (IAS), School of Chemistry and Molecular

Engineering, Jiangsu National Synergetic Innovation Center for Advanced Materials,

Nanjing Tech University, Nanjing 211816, China

*To whom correspondence should be addressed.

E-mail: ias_jhuang@njtech.edu.cn

E-mail: ias_hxu@njtech.edu.cn

\section{Content for Supporting Information:}

1. Materials.

2. Syntheses and Characterization of Compounds.

3. Supplementary Figures and Tables.

\section{Materials}

Tetrakis(triphenylphosphine)palladium (99.5\%), anhydrous potassium carbonate (98\%) and phenylboronic acid (98\%) were purchased from Alfa Aesar. $p$-tolylboronic acid (97\%) and 1,4-phenylenediboronic acid (98\%) were purchased from J\&K Scientific Ltd. Monomer M2 was purchased from Derthon Optoelectronic Materials Science Technology Co. LTD (Shenzhen, China). Monomer M1 ${ }^{[1]}$ and M3 ${ }^{[2]}$ were prepared according reported procedures. A amphiphilic diblock copolymers, polystyrene-b-poly(acrylic acid) (PS- $b$-PAA, PS chain $\mathrm{M}_{\mathrm{n}}=16000 \mathrm{Da}$, PAA chain $\mathrm{M}_{\mathrm{n}}=3500 \mathrm{Da}$, polydispersity 1.2) was purchased from Polymer Source Inc (Quebec, Canada). All the reactions were carried out under nitrogen atmosphere. All organic solvents were purchased from commercial sources. 


\section{Syntheses and Characterization of Compounds}

General Procedure for the Synthesis of TTFT and DTFT (Figure S1). M1 (58.4 $\mathrm{mg}, 0.10 \mathrm{mmol})$ or $\mathrm{M} 3(63.3 \mathrm{mg}, 0.10 \mathrm{mmol})$, M2 (64.5 $\mathrm{mg}, 0.10 \mathrm{mmol})$, tetrakis(triphenylphosphine)palladium $(2 \mathrm{mg})$, tetrahydrofuran $(6 \mathrm{~mL})$ and $2 \mathrm{~mL}$ of $\mathrm{K}_{2} \mathrm{CO}_{3}(2 \mathrm{~mol} / \mathrm{L})$ were added into a Schlenk flask in a nitrogen-filled glovebox. After stirring at $60{ }^{\circ} \mathrm{C}$ for $72 \mathrm{~h}$, the mixture was filtered and then was precipitated into methanol. The solid was filtered and washed in a Soxhlet extractor with methanol followed by acetone to remove residual catalyst and low-molecular-weight material.

Monomer M1. The yield was $67 \%$ as a white solid. ${ }^{1} \mathrm{H}$ NMR $\left(400 \mathrm{MHz}, \mathrm{CDCl}_{3}, \delta\right)$ : 7.53-7.50 (m, 4H), 7.08-7.06 (m, 6H), 7.03-6.97 (m, 8H), 1.32-1.30 (s, 24H, cis-4H and trans-4H overlapped). ${ }^{13} \mathrm{C} \mathrm{NMR}\left(400 \mathrm{MHz}, \mathrm{CDCl}_{3}, \delta\right): 146.83,146.66,143.63$, $143.45,141.38,134.26,134.15,131.47,130.79,127.82,127.71,126.64,126.57$, 83.76, 24.96 .

Monomer M3. The yield was $67 \%$ as a yellow solid. ${ }^{1} \mathrm{H}$ NMR (400 $\mathrm{MHz}, \mathrm{CDCl}_{3}, \delta$ ): $8.38(\mathrm{~m}, 4 \mathrm{H}, \mathrm{Ar}), 7.98(\mathrm{~d}, J=17.6 \mathrm{~Hz}, 2 \mathrm{H}, \mathrm{CH}=\mathrm{CH}), 7.91(\mathrm{~d}, J=8.0 \mathrm{~Hz}, 4 \mathrm{H}, \mathrm{Ar})$, $7.68(\mathrm{~d}, J=8.0 \mathrm{~Hz}, 4 \mathrm{H}, \mathrm{Ar}), 7.47$ (m, 4H, Ar), 6.95 (d, 2H, $J=16.4 \mathrm{~Hz}, \mathrm{CH}=\mathrm{CH})$, $1.41\left(\mathrm{~s}, 24 \mathrm{H},-\mathrm{CH}_{3}\right) .{ }^{13} \mathrm{C} \mathrm{NMR}\left(400 \mathrm{MHz}, \mathrm{CDCl}_{3}, \delta\right): 140.02,137.58,135.42,132.73$, $129.62,126.56,126.34,125.98,125.43,84.02,24.94$.

TTFT: The yield was $43 \%$ as a deep red solid. $\mathrm{M}_{\mathrm{w}}=5.67 \times 10^{4}, \mathrm{M}_{\mathrm{n}}=2.15 \times 10^{4}$, $\mathrm{PDI}=2.64$, (GPC, polystyrene); ${ }^{1} \mathrm{H} \mathrm{NMR}\left(400 \mathrm{MHz}, \mathrm{CDCl}_{3}, \delta\right.$ ): 8.12 (br, Ar-H), 7.99 (br, Ar-H), 7.74 (br, Ar-H), 7.31 (br, Ar-H), 7.16 (br, Ar-H), 2.71 (br, $\mathrm{CH}_{2}$ ), 1.56-1.67 (br, $\left.\mathrm{CH}_{2}\right), 0.89$ (br, $\mathrm{CH}_{3}$ )

DTFT: The yield was $57 \%$ as a deep red solid. $\mathrm{M}_{\mathrm{w}}=4.16 \times 10^{4}, \mathrm{M}_{\mathrm{n}}=1.52 \times 10^{4}$, $\mathrm{PDI}=2.74$ (GPC, polystyrene); ${ }^{1} \mathrm{H}$ NMR $\left(400 \mathrm{MHz}, \mathrm{CDCl}_{3}, d\right): 8.44$ (br, Ar-H), 8.03 (br, $\mathrm{CH}=\mathrm{CH}$ ), 7.80-7.52 (br, Ar-H), 7.33 (br, Ar-H), 7.01 (br, $\mathrm{CH}=\mathrm{CH}), 2.75$ (br, $\mathrm{CH}_{2}$ ), 1.34-1.73 (br, $\left.\mathrm{CH}_{2}\right), 0.91$ (br, $\mathrm{CH}_{3}$ )

\section{Supplementary Figures and Tables}



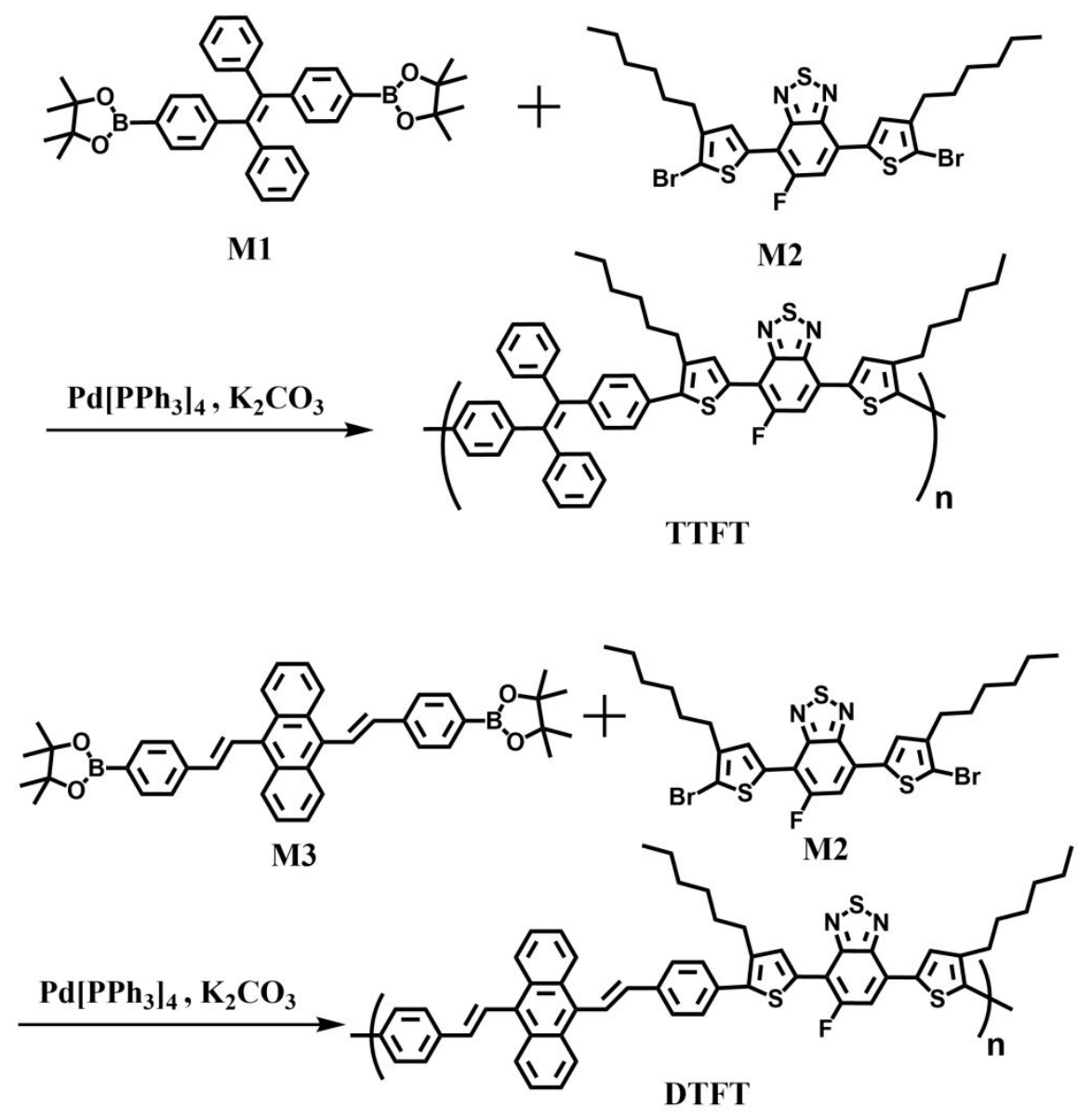

Figure S1. Synthesis of TTFT and DTFT.

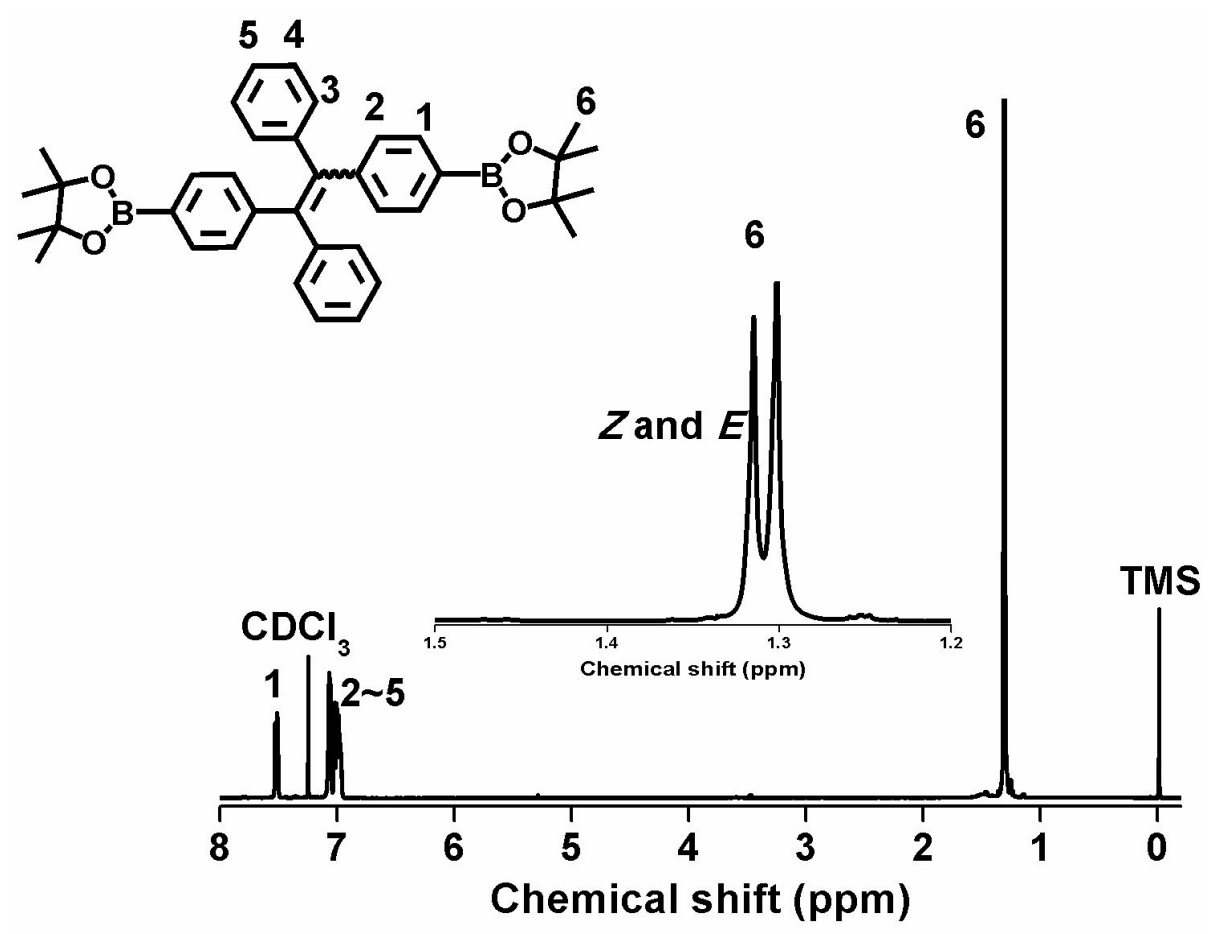

Figure S2. The ${ }^{1} \mathrm{H}$ NMR spectra of M1 $\left(400 \mathrm{MHz}, \mathrm{CDCl}_{3}\right)$. 


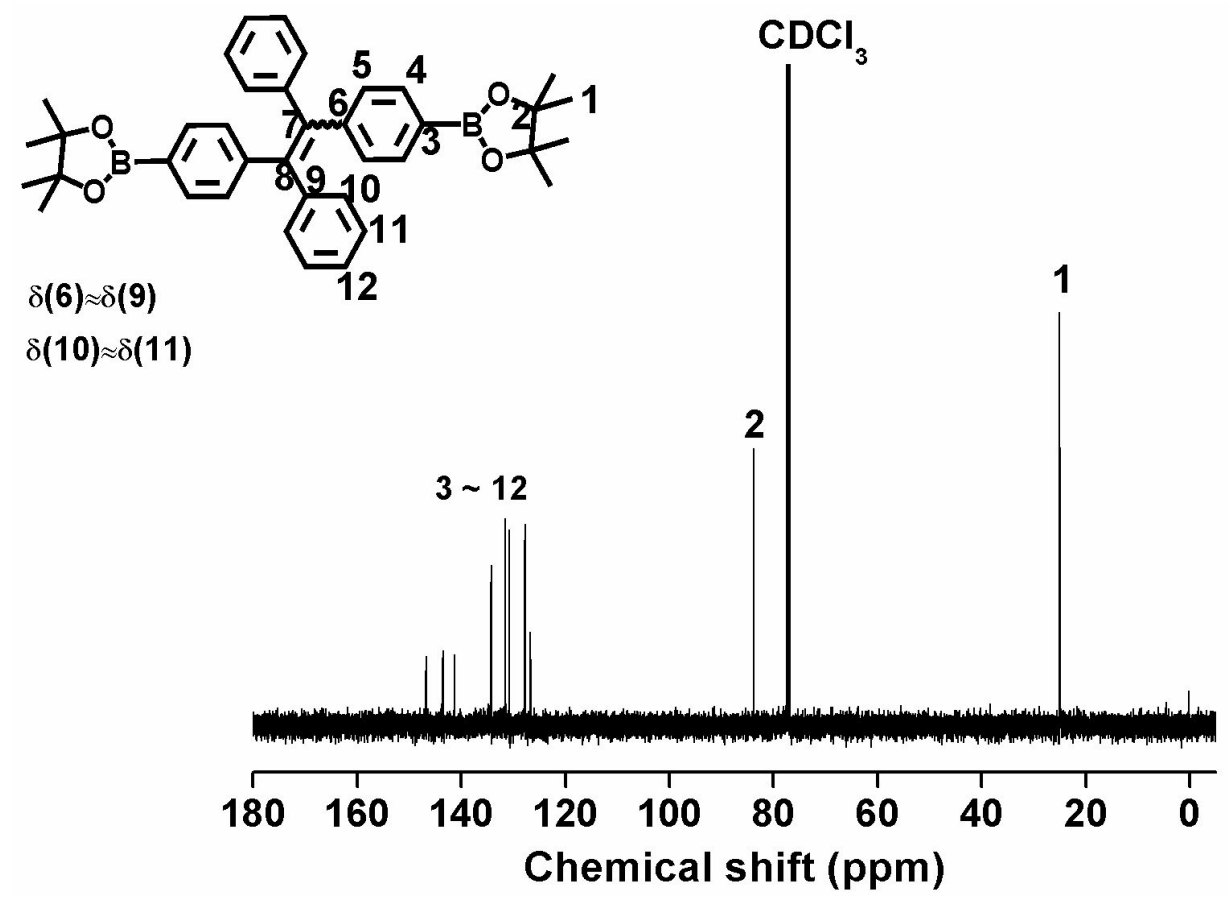

Figure S3. The ${ }^{13} \mathrm{C}$ NMR spectra of $\mathrm{M} 1\left(400 \mathrm{MHz}, \mathrm{CDCl}_{3}\right)$.
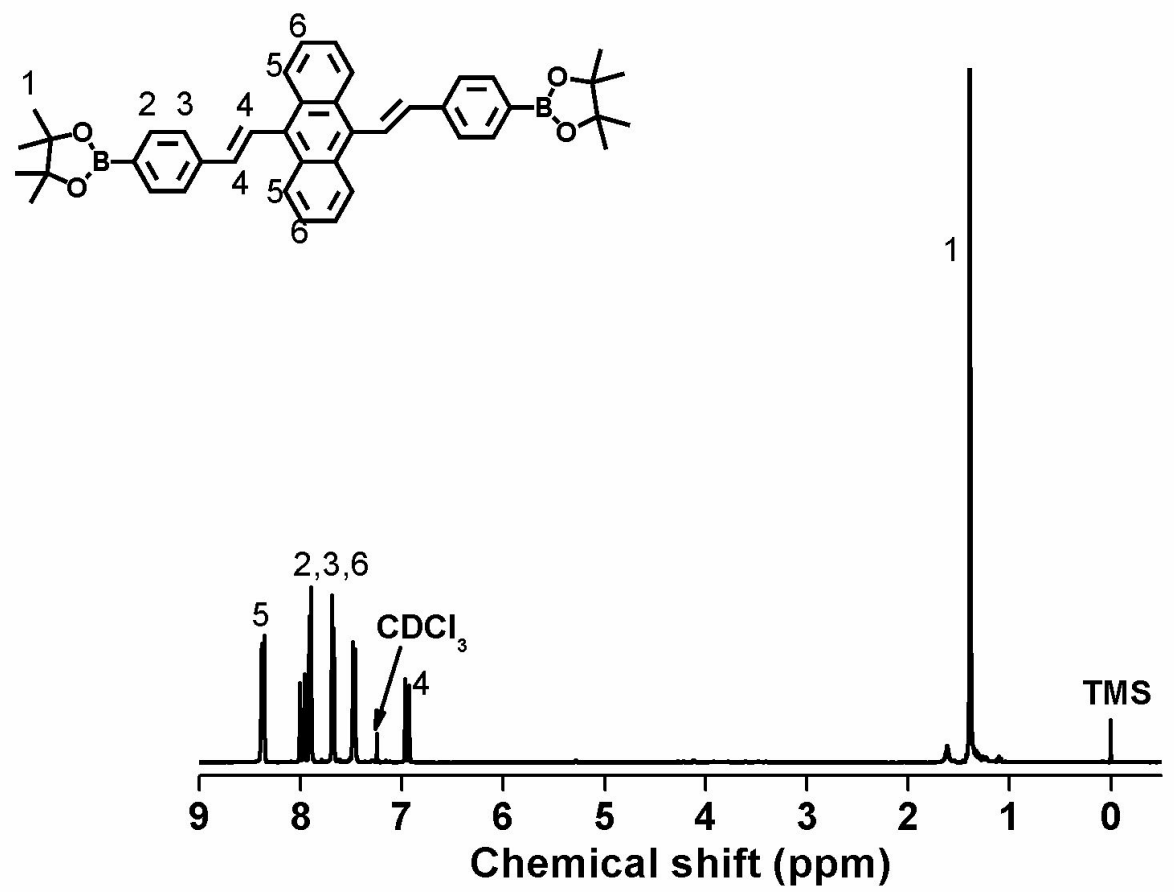

Figure S4. The ${ }^{1} \mathrm{H}$ NMR spectra of $\mathrm{M} 3\left(400 \mathrm{MHz}, \mathrm{CDCl}_{3}\right)$. 

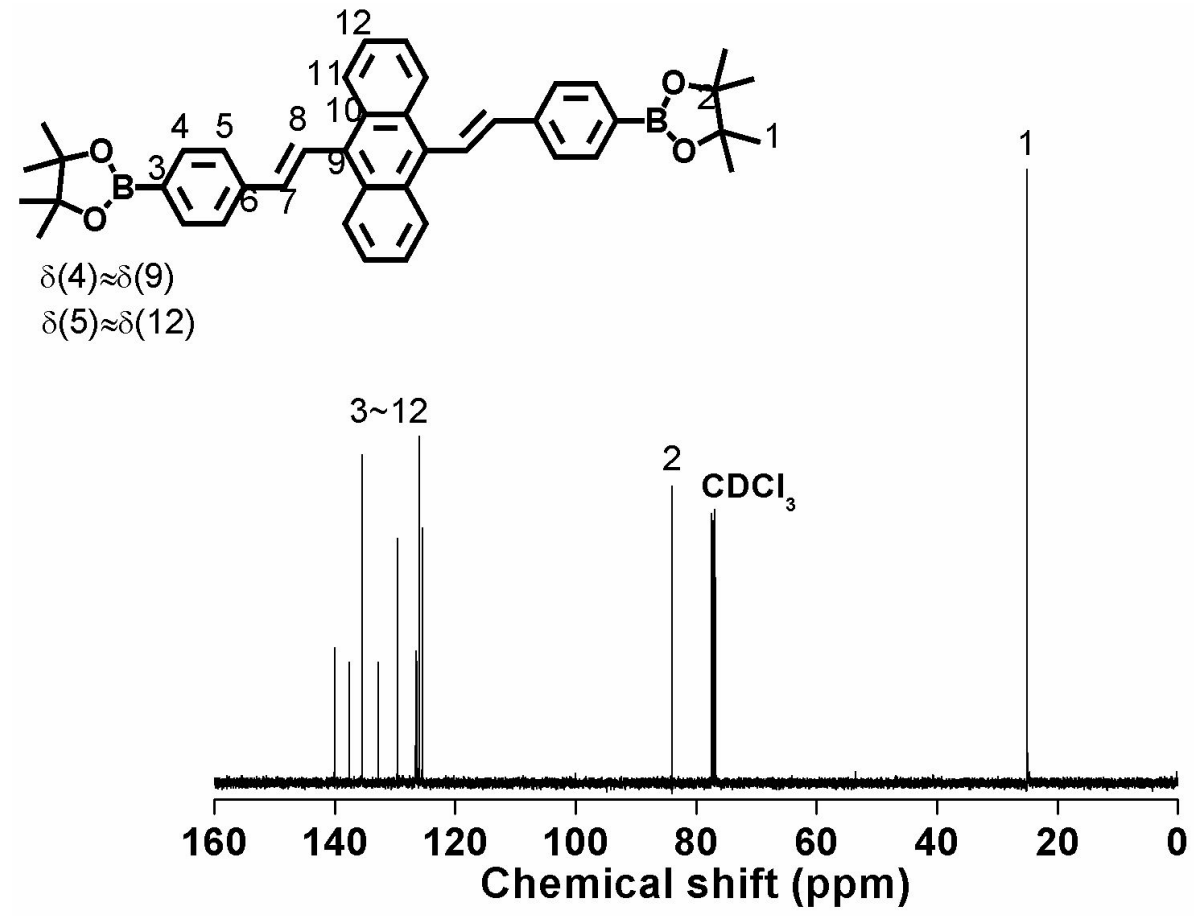

Figure S5. The ${ }^{13} \mathrm{C}$ NMR spectra of $\mathrm{M} 3\left(400 \mathrm{MHz}, \mathrm{CDCl}_{3}\right)$.

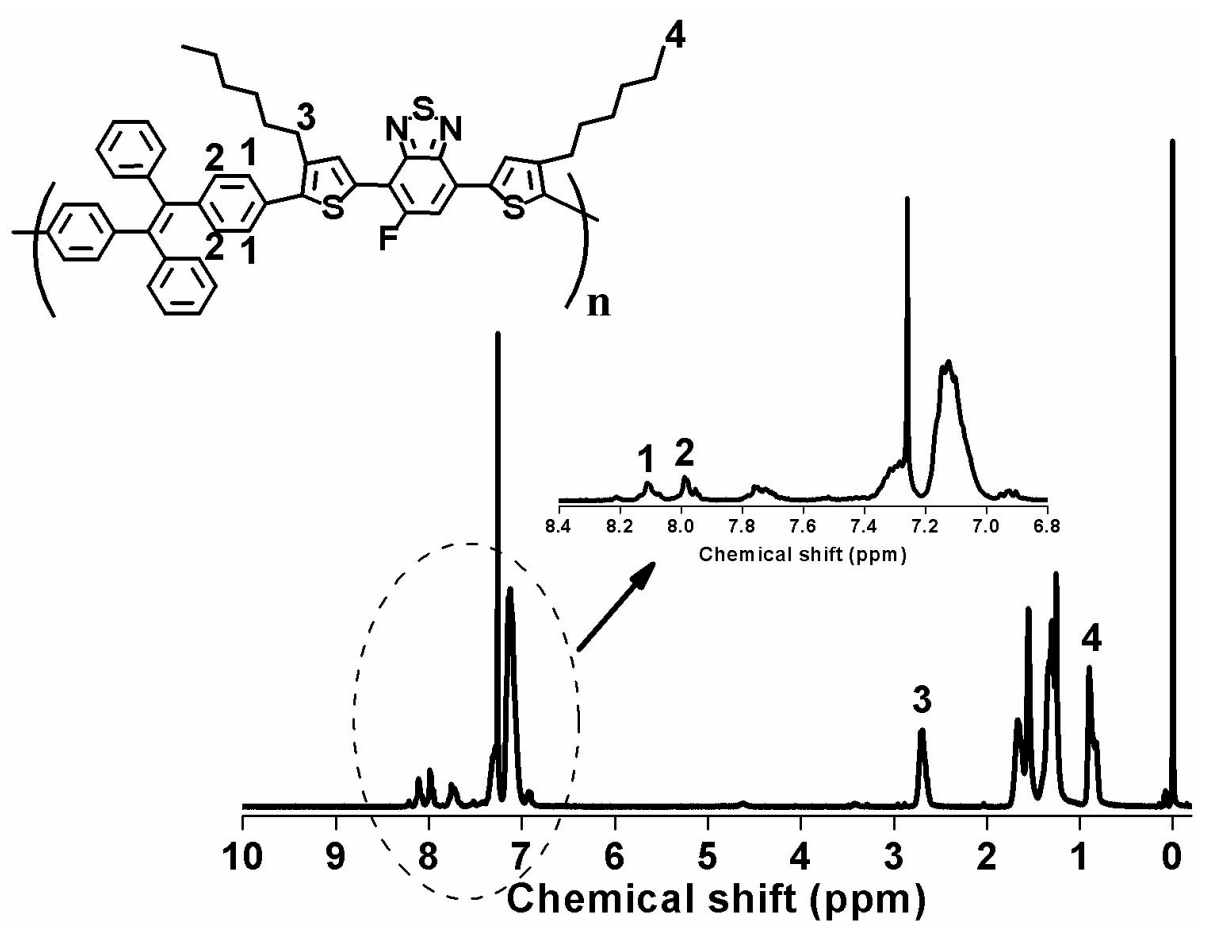

Figure S6. The ${ }^{1} \mathrm{H}$ NMR spectra of TTFT $\left(400 \mathrm{MHz}, \mathrm{CDCl}_{3}\right)$. 


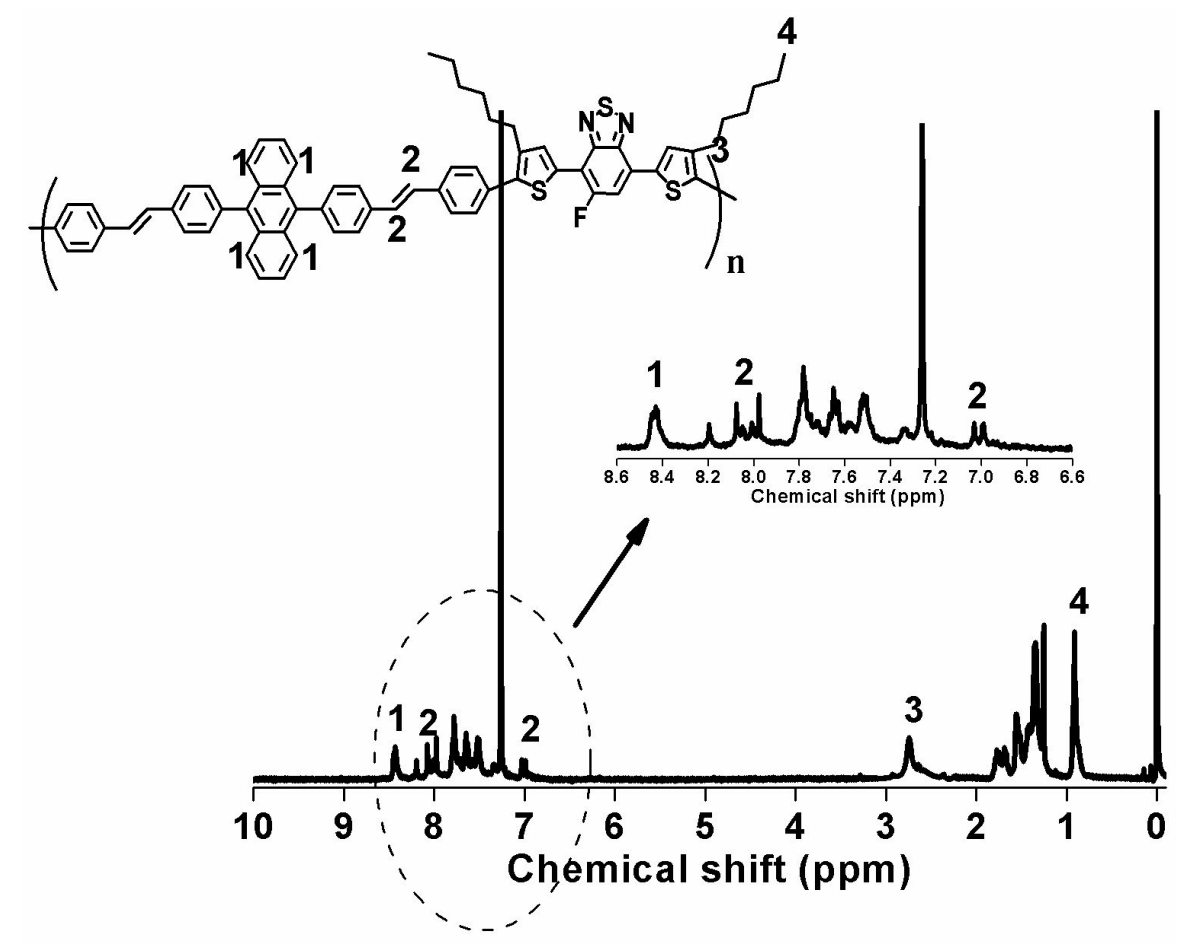

Figure S7. The ${ }^{1} \mathrm{H}$ NMR spectra of DTFT (400 MHz, $\left.\mathrm{CDCl}_{3}\right)$.

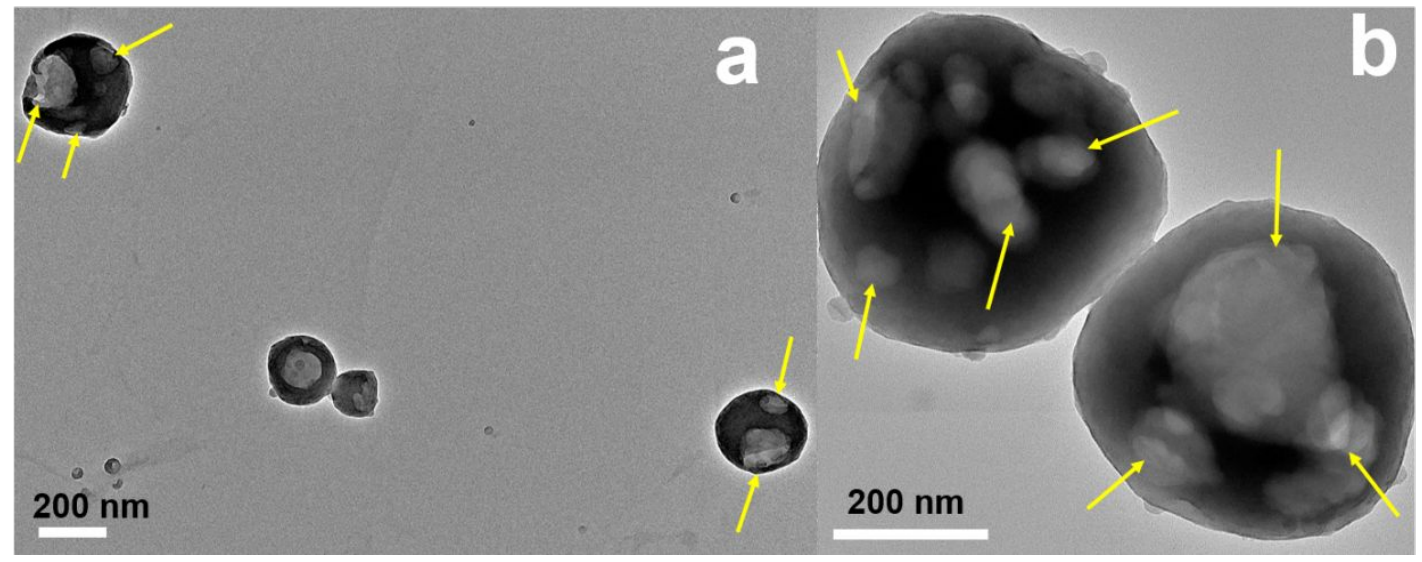

Figure S8 TEM images of assembling products with the mass concentration ratios TTFT:PS- $b$-PAA:PBA = 3:1:12 (a) and DTFT:PS- $b$-PAA:PBA = 3:1:12 (b). 


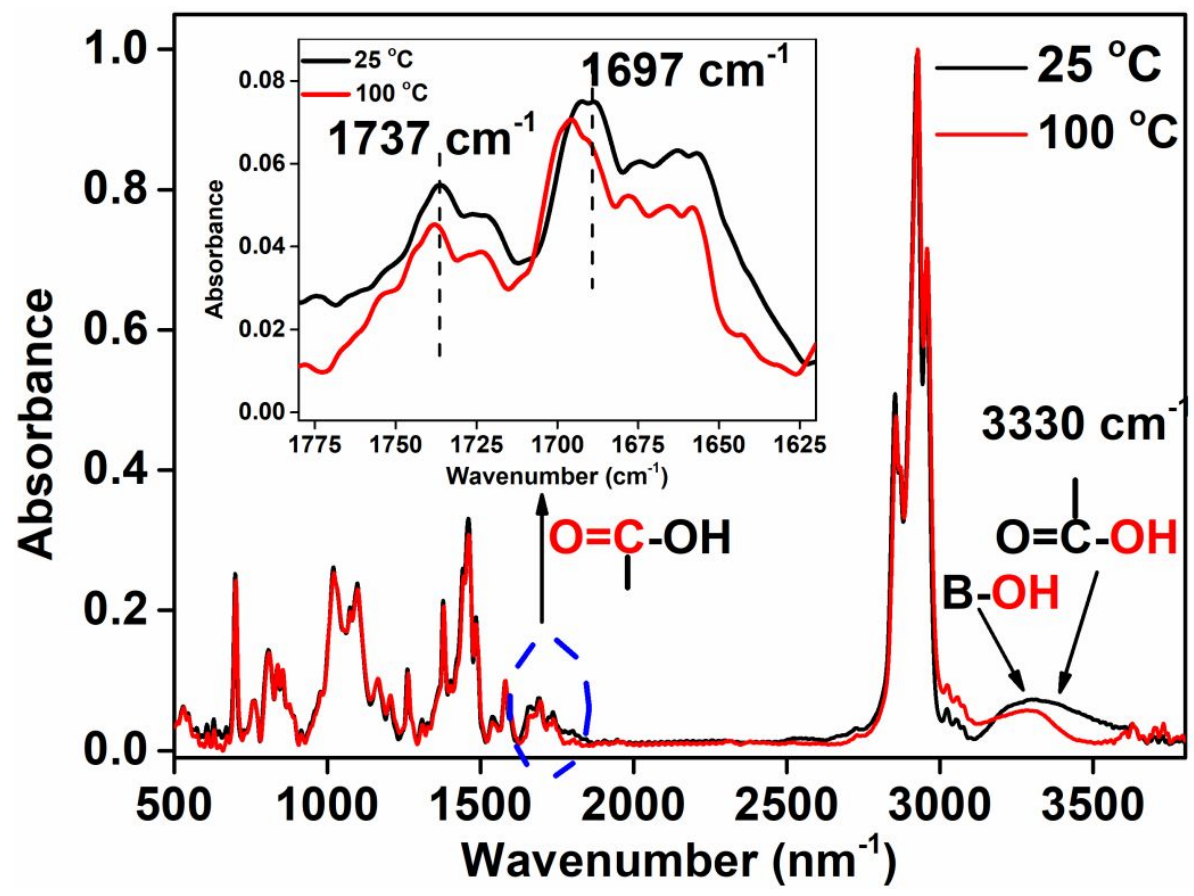

Figure S9 Variable-temperature FT-IR absorption spectra of the change of CPNBs-311T at $25{ }^{\circ} \mathrm{C}$ and $100{ }^{\circ} \mathrm{C}$; Inserted Figure: regionally amplified variable-temperature FT-IR absorption spectra of carbonyl peak.

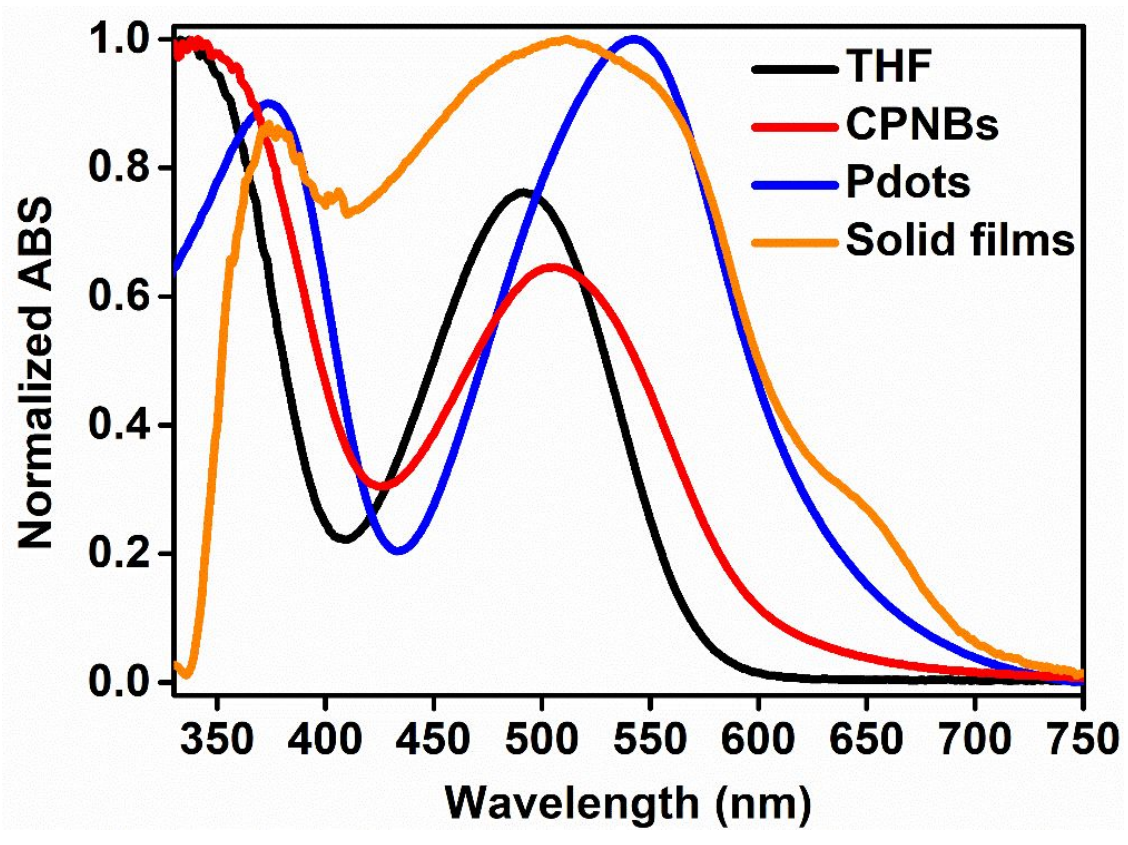

Figure S10 Normalized UV-vis spectra of TTFT dilute solution $(0.01 \mathrm{mg} / \mathrm{mL}$ in THF), TTFT solid film, Pdots-T, CPNBs-311T. 


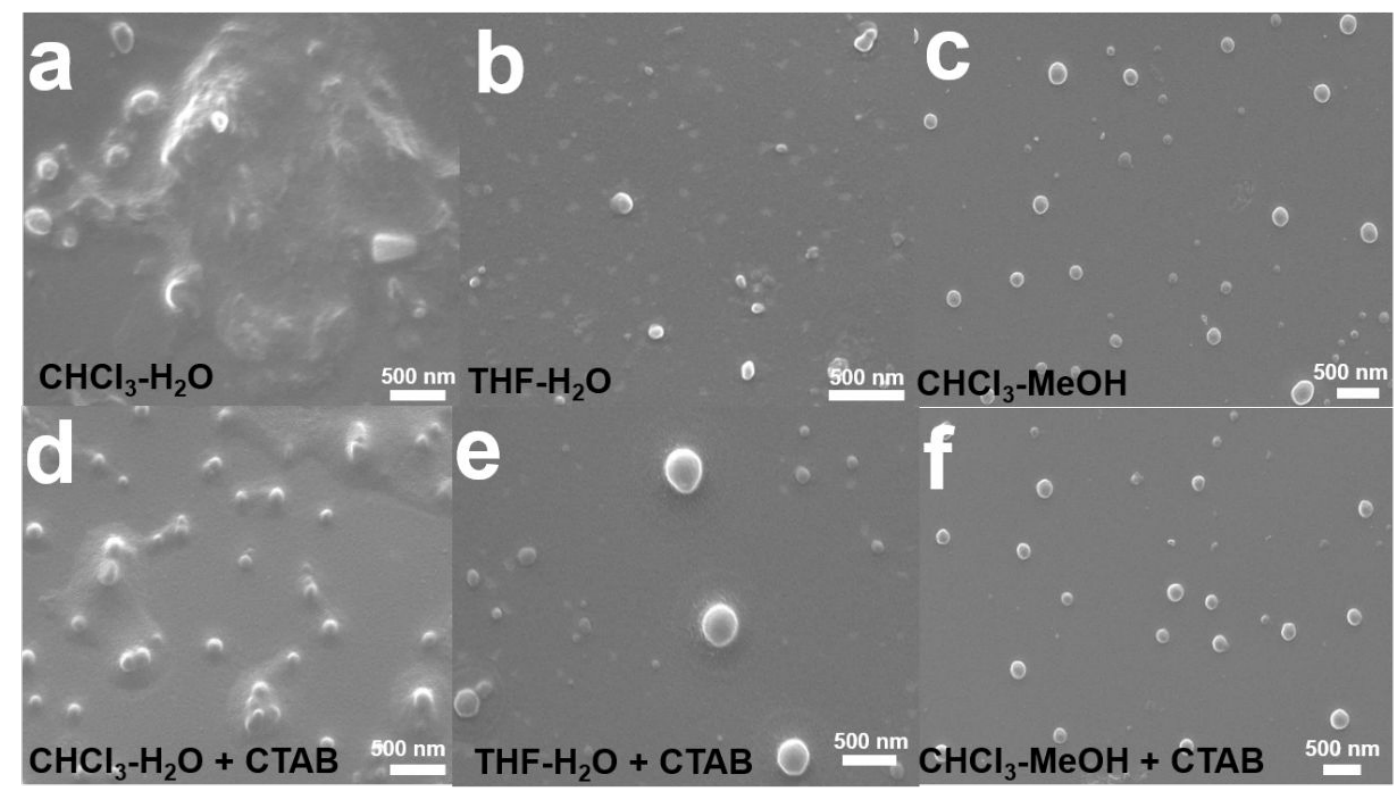

Figure S11. The SEM images of assembling morphology of TTFT in different mixed solvent system $($ scale bar $=500 \mathrm{~nm})$.

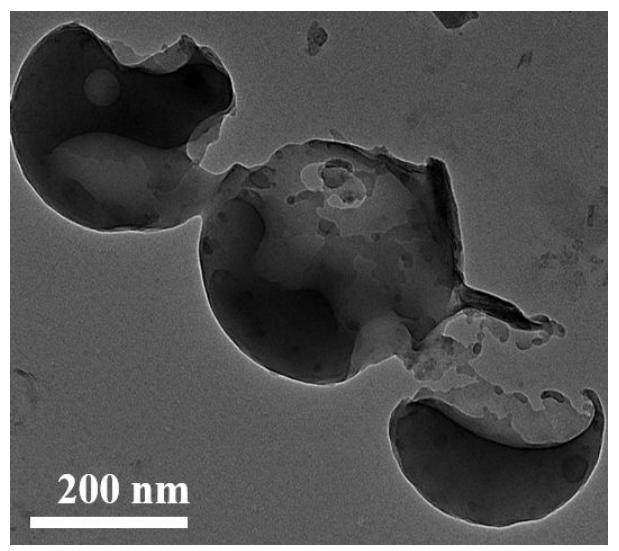

Figure S12 TEM images of products after adding $1 \mathrm{~mol} / \mathrm{L} \mathrm{NaOH}$ into CPNBs-311T solution $(\mathrm{pH}$ is $\sim 11)$. 


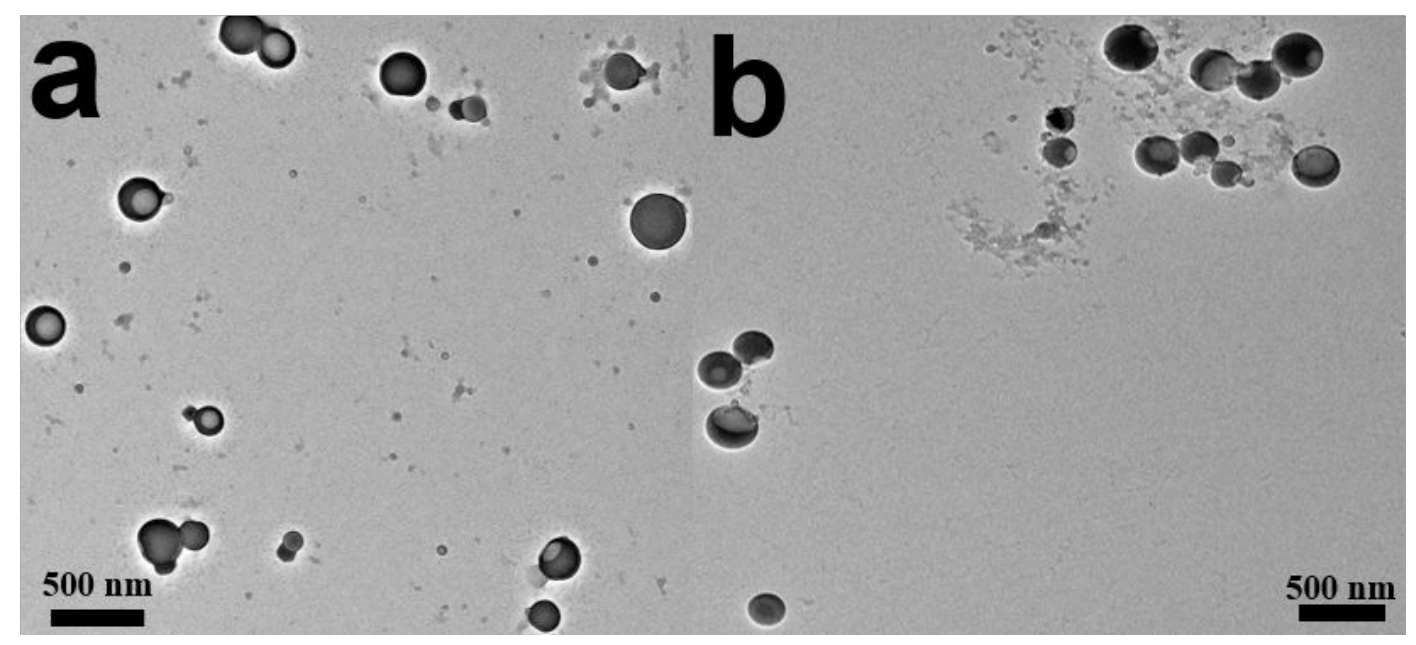

Figure S13 TEM images of assembling products with the mass concentration ratios

TTFT:PS- $b$-PAA: $p$-tolylboronic acid $=3: 1: 1$ (a) and TTFT:PS- $b$-PAA:

1,4-phenylenediboronic acid $=3: 1: 1(\mathrm{~b})$. 

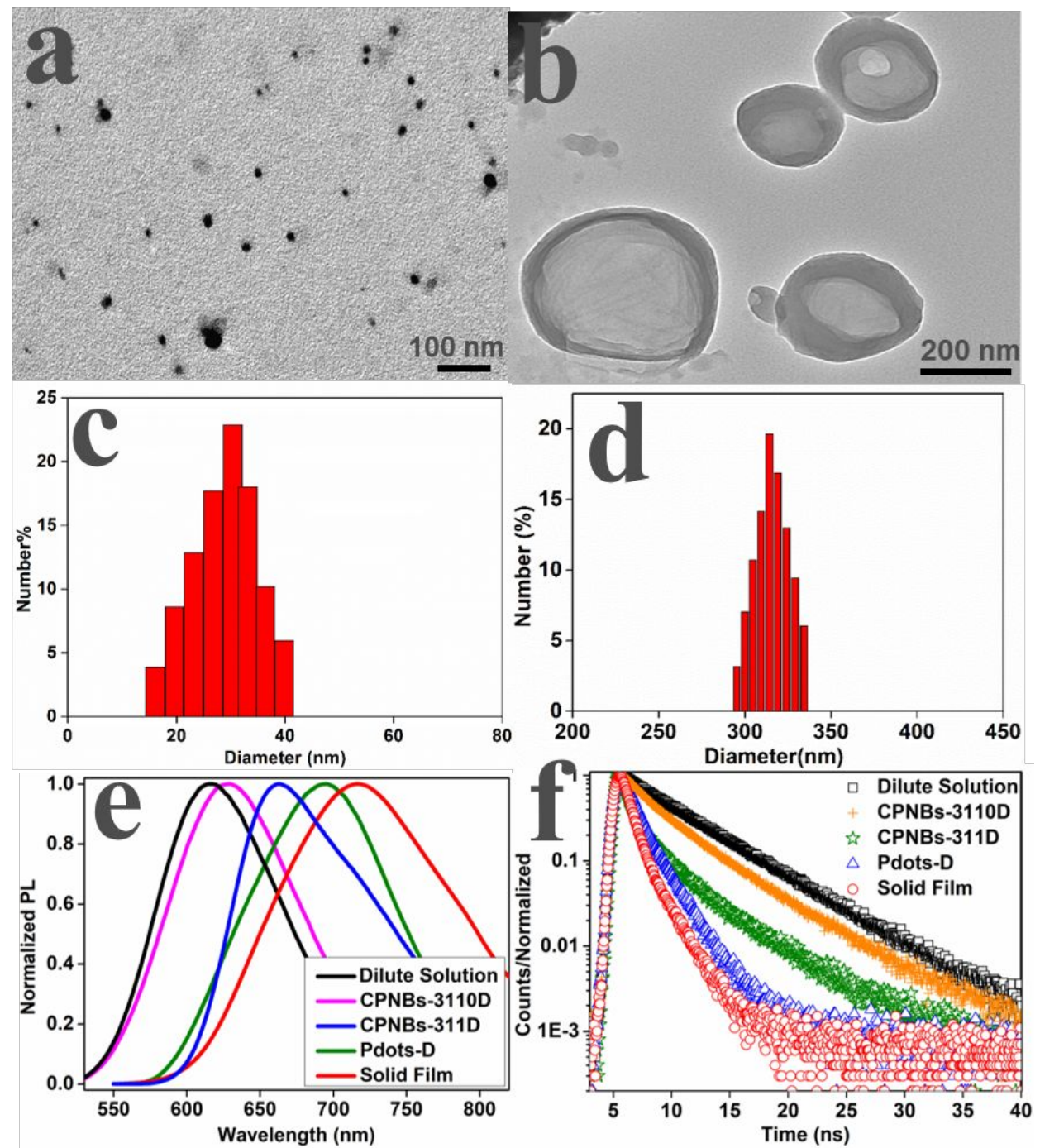

Figure S14. TEM images of Pdots-T (a) (scale bar $=100 \mathrm{~nm}$ ), CPNBs-3110D (b) (scale bar $=200 \mathrm{~nm}$ ); Hydrodynamic diameter of Pdots-D (c), CPNBs-3110D (d) measured by DLS; (e) Normalized emission spectra of dilute solution $(0.01 \mathrm{mg} / \mathrm{mL}$ in THF), solid film, Pdots-D, CPNBs-311D and CPNBs-3110D; (f) Time-resolved fluorescence decay curves at $480 \mathrm{~nm}$ for dilute solution $(0.01 \mathrm{mg} / \mathrm{mL}$ in THF), solid film, Pdots-D, CPNBs-311D and CPNBs-3110D. 
Table S1. The mass concentrations of ingredients in THF for the assembly system based on DTFT series.

\begin{tabular}{lccc}
\hline & & & phenylboronic \\
Sample & DTFT & PS-PAA & acid \\
& $(\mu \mathrm{g} / \mathrm{mL})$ & $(\mu \mathrm{g} / \mathrm{mL})$ & $(\mu \mathrm{g} / \mathrm{mL})$ \\
\hline Pdots-D & 150 & 50 & 0 \\
CPNBs-311D & 150 & 50 & 50 \\
CPNBs-3110D & 150 & 50 & 500 \\
\hline
\end{tabular}

Table S2. Fluorescence properties under different aggregation states based on DTFT series

\begin{tabular}{|c|c|c|c|}
\hline Sample & $\begin{array}{c}{ }^{\mathrm{a}} \text { Maximum } \\
\text { Emission Peak } \\
(\mathrm{nm})\end{array}$ & $\begin{array}{c}{ }^{\mathrm{b}} \text { AFQY } \\
(\%)\end{array}$ & $\begin{array}{c}{ }^{\mathrm{c}} \text { Average } \\
\text { Fluorescence } \\
\text { Lifetime } \tau_{a}(\mathrm{~ns})\end{array}$ \\
\hline $\begin{array}{l}\text { DTFT }(0.01 \\
\mathrm{mg} / \mathrm{mL} \text { in THF })\end{array}$ & 618 & 27.1 & 5.09 \\
\hline CPNBs-3110D & 630 & 27.8 & 3.95 \\
\hline CPNBs-311D & 661 & 29.6 & 1.61 \\
\hline Pdots-D & 695 & 31.2 & 1.26 \\
\hline Solid Films & 716 & 37.7 & 0.95 \\
\hline \multicolumn{4}{|c|}{$\begin{array}{l}\text { Instrument FLS } 980 \text { and excitation wavelength was } 480 \mathrm{~nm} \text {; } \\
\text { Absolute fluorescence quantum yield (AFQY) were determined by } \\
\text { using an integrating sphere F-M01 on the Edinburgh Instrument } \\
\text { FLS980; }{ }^{\mathrm{b}} \text { Average Fluorescence Lifetime } \tau_{a}(\mathrm{~ns}) \text { was calculated by } \\
\text { the equation } \tau_{a}=\mathrm{A}_{1} \tau_{1}+\mathrm{A}_{2} \tau_{2}, \mathrm{~A}_{1} \text { and } \mathrm{A}_{2} \text { is the percentage of } \\
\text { fluorescence lifetime } \tau_{1} \text { and } \tau_{2} \text { respectively. } \tau_{1}, \tau_{2}, \mathrm{~A}_{1} \text { and } \mathrm{A}_{2} \text { were } \\
\text { measured by Edinburgh Instrument FLS980, a picosecond pulsed } \\
\text { diode laser EI-EPL- } 485 \text { as excitation light source, excitation } \\
\text { wavelength was } 485 \mathrm{~nm} \text {, pulse width } 80 \text { picoseconds, frequency } \\
10 \mathrm{MHz} \text {. }\end{array}$} \\
\hline
\end{tabular}




\section{REFERENCES}

[1] Yu, C. -Y.; Hsu, C. -C., Synthesis, characterization and aggregation-induced emission of alternating copolymers containing cyclophanes and tetraphenylethenes. Polymer 2018, 137, 30-37.

[2] Wu, D. -E.; Wang, M. -N.; Luo, Y. -H.; Wen, G. -J.; Sun, B. -W., Influence of halogen atoms on the structures and photophysical properties of 9,10-distyrylanthracene (DSA). CrystEngComm 2015, 17, 9228-9239. 